\title{
Salvianolic acid B alleviates myocardial ischemic injury by promoting mitophagy and inhibiting activation of the NLRP3 inflammasome
}

\author{
YANG HU* ${ }^{*}$, XINYU WANG* ${ }^{*}$ QINGJU LI, YUNZHENG PAN and LI XU \\ Jiangsu Key Laboratory for Pharmacology and Safety Evaluation of Chinese Materia Medica, \\ School of Pharmacy, Nanjing University of Chinese Medicine, Nanjing, Jiangsu 210023, P.R. China
}

Received February 14, 2020; Accepted September 22, 2020

DOI: $10.3892 / \mathrm{mmr} .2020 .11589$

\begin{abstract}
Ischemic heart disease is a major cause of mortality and disability worldwide. Salvianolic acid B (Sal B) is one of the main water-soluble components of Salvia miltiorrhiza Bge. Numerous studies have demonstrated that Sal B could exert significant anti-inflammatory and cardiovascular protective effects; however, the underlying mechanisms remain unclear. To elucidate the association between myocardial ischemia and inflammation, and to develop effective protective drugs, a rat model of myocardial ischemia was induced using isoproterenol (ISO) and an inflammation model in H9C2 cells was induced with lipopolysaccharide + adenosine triphosphate. Both of these models were treated with different concentrations of Sal B $(5,10$ and $15 \mathrm{mg} / \mathrm{kg}$ in vivo; 1 , 5 and $25 \mu \mathrm{M}$ in vitro). In vivo, the serum levels of creatine kinase isoenzyme MB, glutamic oxaloacetic transaminase and IL-1 $\beta$, the cardiac function and the mRNA expression levels of NLR family pyrin domain-containing 3 (NLRP3) inflammasome components were evaluated using ELISAs, an electrocardiogram, hematoxylin and eosin staining and reverse transcription-quantitative PCR, respectively. The results demonstrated that treatment with Sal B markedly alleviated the acute myocardial ischemic injury induced by hypodermic injection of ISO in rats. In vitro, the results of reactive oxygen species (ROS) detection, JC-1 staining, western blotting and TUNEL assays showed that Sal B treatment significantly inhibited intracellular ROS production, increased the mitochondrial membrane potential, regulated the expression
\end{abstract}

Correspondence to: Professor Li Xu, Jiangsu Key Laboratory for Pharmacology and Safety Evaluation of Chinese Materia Medica, School of Pharmacy, Nanjing University of Chinese Medicine, 138 Xianlin Avenue, Qixia, Nanjing, Jiangsu 210023, P.R. China E-mail: xuli64@163.com

*Contributed equally

Key words: salvianolic acid B, NLR family pyrin domain-containing 3 inflammasome, mitophagy, myocardial ischemia of mitophagy-related proteins, inhibited the activation of the NLRP3 inflammasome and inhibited apoptosis in H9C2 cells. In conclusion, these findings indicated that Sal B exerted protective effects against myocardial ischemic injury by promoting mitophagy and maintaining mitochondrial function.

\section{Introduction}

Cardiovascular disease is a major health issue worldwide, with acute myocardial ischemia accounting for $>40 \%$ deaths in China (1). Severe myocardial ischemia may result in a characteristic pattern of metabolic and ultrastructural changes (2), which can lead to irreversible injury. Although several studies have been performed, the molecular mechanisms underlying myocardial ischemic injury remain unclear $(3,4)$. In addition, it has been reported that the occurrence of myocardial ischemia is often accompanied by severe inflammatory response (5) and activation of the NLR family pyrin domain-containing 3 (NLRP3) inflammasome (6).

The NLRP3 inflammasome is a cytosolic protein complex comprising NLRP3, apoptosis-associated speck-like protein (ASC) and caspase-1. Notably, the NLRP3 inflammasome is assembled in response to several endogenous "danger signals", such as oxidative stress, lysosome destabilization and mitochondrial dysfunction (7). Recently, it has been reported that activation of the NLRP3 inflammasome is associated with the pathogenesis of numerous inflammatory diseases $(8,9)$, and that it may regulate the secretion of several pro-inflammatory cytokines, such as IL-1 $\beta$ and IL-18 (10). Furthermore, inhibition of the NLRP3 signaling pathway has been shown to protect against myocardial ischemic injury induced by coronary artery ligation (11).

Sirtuin 1 (SIRT1) is a member of the sirtuin class III histone deacetylase family. It serves important roles in several important biological processes, including mitophagy, cell senescence and cell apoptosis (12). In recent years, several studies have focused on the role of SIRT1-mediated mitophagy in the regulation of NLRP3 activation. These studies suggested that SIRT1-mediated mitophagy is a negative regulator of NLRP3 inflammasome activation (13) and could significantly protect against myocardial ischemic injury (14). However, 
whether salvianolic acid B (Sal B) could alleviate myocardial ischemic injury by promoting SIRT1-mediated mitophagy remains unclear.

Salvia miltiorrhiza Bge has been used to treat myocardial ischemia in mainland China for $>2,000$ years. Compared with chemical drugs, this plant has numerous advantages, including multi-ingredients (which enables less opportunity for resistance), multi-targets and few side effects $(15,16)$. As the main active water-soluble substance in S. miltiorrhiza Bge, Sal B has been reported to exert several pharmacological activities, including antitumor (17), anti-inflammatory (18) and antioxidant (19) effects. It has previously been reported that Sal B may exert protective effects against myocardial ischemic injury (20). However, whether Sal B could regulate mitophagy and inhibit activation of the NLRP3 inflammasome during myocardial ischemic injury remains unclear. Therefore, the present study investigated whether Sal B could alleviate myocardial ischemic injury by promoting cell mitophagy and inactivating the NLRP3 inflammasome.

\section{Materials and methods}

Materials. Sal B was from Shanghai Yuanye Biotechnology (cat. no. B20261). Isoproterenol (ISO) was obtained from Beijing Solarbio Science \& Technology Co., Ltd. (cat. no. II0200). Dulbecco's modified Eagle's medium (DMEM) containing high glucose was obtained from Wisent Biotechnology. Trypsin was obtained from Beyotime Institute of Biotechnology. The creatine kinase isoenzyme MB (CK-MB; cat. no. H197) and glutamic oxaloacetic transaminase (GOT; cat. no. C010-2-1) ELISA kits were obtained from Nanjing Jiancheng Bioengineering Institute. Evagreen $2 X$ quantitative PCR (qPCR) MasterMix-Low Rox kit and the IL-1 $\beta$ ELISA kit (cat. no. K4796-100) were purchased from Abmgoodchina, Inc. and BioVision, Inc., respectively. Adenosine triphosphate (ATP) was obtained from Amresco, LLC. Lipopolysaccharide (LPS) was obtained from Cell Signaling Technology, Inc. Primary antibodies against SIRT1 (cat. no. ab189494), microtubule-associated protein 1A/1B-light chain 3 (LC3; cat. no. ab48394), P62 (cat. no. ab91526), PTEN-induced kinase 1 (PINK1; cat. no. ab23707), Beclin-1 (cat. no. ab62557), NLRP3 (cat. no. ab214185), IL-1 $\beta$ (cat. no. ab205924) were purchased from Abcam, and primary antibodies against Parkin (cat. no. 14060-1-AP), caspase-1 (cat. no. 22915-1-AP) and GAPDH (cat. no. 10494-1-AP) were purchased from Proteintech Group, Inc. The distilled water used in this study was purified using a Milli-Q system (EMD Millipore). All culture plates were purchased from Corning, Inc.

Animals. Male Sprague-Dawley (SD) rats (age, 2 months; weight, $180-220 \mathrm{~g}$ ), were housed in pathogen-free conditions under a 12 -h light/dark cycle at $22-24^{\circ} \mathrm{C}$ (humidity, $40-70 \%$ ) with ad libitum access to a normal pellet diet and clean water. The protocol of the present study was approved by the Animal Ethics Committee of Nanjing University of Chinese Medicine (Nanjing, China; approval no. 201906A036).

A total of 50 male SD rats were randomly divided into the following five groups ( $n=10 /$ group): i) control group; ii) model group; iii) model + Sal B $(5 \mathrm{mg} / \mathrm{kg})$ group; iv) model + Sal B $(10 \mathrm{mg} / \mathrm{kg})$ group; and v) model + Sal B $(15 \mathrm{mg} / \mathrm{kg})$ group. The rats in the Sal B treatment groups received a daily intraperitoneal injection of Sal B (dissolved in PBS) for 7 consecutive days, whereas rats in the control and model groups were treated with equal amounts of normal saline. Furthermore, the acute myocardial ischemia model was induced by hypodermic injection of ISO $(30 \mathrm{mg} / \mathrm{kg} / \mathrm{d})$ once daily from the 5 th day. Notably, the modelling method of ISO-induced myocardial ischemia has previously been confirmed to be effective $(21,22)$. Following treatment with Sal B for $24 \mathrm{~h}$ on the 7th day, all rats were anesthetized by intraperitoneal injection of pentobarbital sodium solution $(50 \mathrm{mg} / \mathrm{kg})$ and a total of $2 \mathrm{ml}$ blood was withdrawn from the abdominal aorta. Subsequently, animals were euthanized with $5 \%$ pentobarbital sodium solution $(150 \mathrm{mg} / \mathrm{kg})$, and the hearts were harvested for a series of pathological and biochemical studies. Euthanasia should result in rapid loss of consciousness, followed by respiratory and cardiac arrest, and ultimate loss of all brain function. Death was confirmed by checking the breathing rate, heartbeat and pupillary reflexes after euthanasia and prior to disposal of the animal.

Determination of ST-segment elevation. Following anesthesia by intraperitoneal injection of $50 \mathrm{mg} / \mathrm{kg}$ sodium pentobarbital, the electrocardiogram (ECG) indexes of rats were determined using a power-lab system (ADInstruments, Ltd.). Myocardial ischemia was verified by ST-segment elevation recorded by ECG and the results were expressed as relative to the control.

Hematoxylin and eosin $(H \& E)$ staining. The heart tissues were fixed in $10 \%$ neutral buffered formalin for $24 \mathrm{~h}$ at room temperature, embedded in paraffin and were then sliced into $5-\mu \mathrm{m}$ conventional sections using a microtome. Subsequently, the embedded blocks were prepared into pathological sections. The sections were stained with H\&E for $5 \mathrm{~min}$ at room temperature. The sections were then mounted and histologically observed under a light microscope (magnification, x200).

Determination of serum CK-MB, GOT and IL-1 $\beta$ concentrations. Blood samples were collected from the abdominal aorta of each rat $24 \mathrm{~h}$ after Sal B administration on the 7th day and the serum was obtained by centrifugation at $1,500 \mathrm{x}$ g for $10 \mathrm{~min}$ at room temperature. Subsequently, the serum levels of CK-MB, GOT and IL-1 $\beta$ were determined using a microplate system (BioTek Instruments, Inc.) and the aforementioned ELISA kits, according to the manufacturer's instructions.

Reverse transcription (RT)-qPCR. Total RNA was extracted from heart tissues using TRIzol ${ }^{\circledR}$ reagent (Invitrogen; Thermo Fisher Scientific, Inc.) and RNA concentration was determined using a NanoDrop (NanoDrop Technologies; Thermo Fisher Scientific, Inc.). Total RNA was reverse transcribed into cDNA using an 5X All-in-one RT MasterMix kit (Abmgoodchina Inc.), according to the manufacturer's instructions. Subsequently, qPCR was performed using the Evagreen 2X qPCR MasterMix-Low Rox kit (Abmgoodchina Inc.) on the ABI 7500 Fast Real-Time PCR Detection system (Applied Biosystems; Thermo Fisher Scientific, Inc.). The following thermocycling conditions were used for the qPCR: Initial denaturation at $95^{\circ} \mathrm{C}$ for $10 \mathrm{~min}$; followed by 40 cycles of $95^{\circ} \mathrm{C}$ for $15 \mathrm{sec}$ and $60^{\circ} \mathrm{C}$ for $60 \mathrm{sec}$. The mRNA expression levels of NLRP3, ASC, caspase-1 and IL-1 $\beta$ were determined 
Table I. Primer sequences used for quantitative PCR.

\begin{tabular}{lll}
\hline Gene & Forward sequence $\left(5^{\prime} \rightarrow 3^{\prime}\right)$ & Reverse sequence $\left(5^{\prime} \rightarrow 3^{\prime}\right)$ \\
\hline NLRP3 & CTCTGCATGCCGTATCTGGT & GTCCTGAGCCATGGAAGCAA \\
Caspase-1 & GACCGAGTGGTTCCCTCAAG & GACGTGTACGAGTGGGTGTT \\
ASC & GGACAGTACCAGGCAGTTCG & GTCACCAAGTAGGGCTGTGT \\
IL-1 $\beta$ & GACTTCACCATGGAACCCGT & GGAGACTGCCCATTCTCGAC \\
GAPDH & GGCAAATTCAACGGCACAGT & AGATGGTGATGGGCTTCCC \\
\hline
\end{tabular}

ASC, apoptosis-associated speck-like protein; NLRP3, NLR family pyrin domain-containing 3.

using the $2^{-\Delta \Delta \mathrm{Cq}}$ method (23) and were normalized to GAPDH expression levels. The sequences of the specific primers used for qPCR analysis are listed in Table I.

Cell culture and treatment. The H9C2 cell line, which is widely used to study myocardial ischemia $(24,25)$, was obtained from the American Type Culture Collection. Cells were cultured in high-glucose DMEM supplemented with $10 \%$ fetal bovine serum (Gibco; Thermo Fisher Scientific, Inc.) at $37^{\circ} \mathrm{C}$ in a $5 \% \mathrm{CO}_{2}$ atmosphere.

H9C2 cells were seeded into a 6-well plate at a density of $5 \times 104$ cells and cultured overnight. Subsequently, the cells were randomly divided into the following five groups: i) Control group; ii) LPS + ATP group; iii) LPS + ATP + Sal B $(1 \mu \mathrm{M})$ group; iv) LPS + ATP + Sal B $(5 \mu \mathrm{M})$ group; and v) LPS + ATP + Sal B $(25 \mu \mathrm{M})$ group. Cells in Sal B treatment groups were pretreated with different concentrations of Sal B $(1,5$ and $25 \mu \mathrm{M})$ for $24 \mathrm{~h}$ at $37^{\circ} \mathrm{C}$. After discarding the supernatant, all cells, except within the control group, were washed twice with PBS and stimulated with LPS $(1 \mu \mathrm{g} / \mathrm{ml})$ for $24 \mathrm{~h}$ at $37^{\circ} \mathrm{C}$. Subsequently, cells were stimulated with ATP $(5 \mathrm{mM})$ for $2 \mathrm{~h}$ at $37^{\circ} \mathrm{C}$. Cells in the control group were cultured normally (high-glucose DMEM supplemented with $10 \% \mathrm{FBS}$ at $37^{\circ} \mathrm{C}$ ). Finally, all cells in all groups were collected for subsequent analyses.

Western blot analysis. Cultured H9C2 cells were lysed in RIPA lysis buffer (Beyotime Institute of Biotechnology) and the total protein concentration was quantified using a BCA assay (Beyotime Institute of Biotechnology). Total protein samples $(50 \mu \mathrm{g})$ were separated by SDS-PAGE on $6-10 \%$ gels. The total protein samples were then electrotransferred onto polyvinylidene fluoride membranes. After blocking with 5\% non-fat milk in TBS- $0.05 \%$ Tween- 20 for $1 \mathrm{~h}$ at room temperature, the membranes were incubated with primary antibodies against NLRP3 (1:1,000), caspase-1 (1:1,000), IL-1 $\beta$ (1:1,000), SIRT1 $(1: 1,000)$, P62 (1:1,000), Parkin $(1: 1,000)$, PINK1 $(1: 1,000)$, Beclin-1 (1:1,000), LC3 (1:1,000) and GAPDH $(1: 5,000)$ at $4^{\circ} \mathrm{C}$ overnight with gentle agitation. Subsequently, the membranes were incubated with corresponding horseradish peroxidase-conjugated secondary antibodies (dilution, 1:1,000; Beyotime Institute of Biotechnology; cat. no. A0208) in non-fat milk for $1 \mathrm{~h}$ at room temperature. The membranes were visualized using an ECL kit (Beyotime Institute of Biotechnology) and densitometric analysis was performed using Image Lab software version 4.0.1 (Bio-Rad Laboratories, Inc.)
Measurement of intracellular reactive oxygen species (ROS) generation. The intracellular ROS levels were measured using 2,7-dichlorofluorescin diacetate (DCFH-DA; cat. no. S0033S; Beyotime Institute of Biotechnology). In the presence of ROS, DCFH-DA is oxidized into fluorescent dichlorofluorescein (DCF) (26). After treatment, the collected cells (1x10\%/well) were resuspended in $1 \mathrm{ml}$ DCFH-DA reagent $(10 \mu \mathrm{M})$ and incubated at $37^{\circ} \mathrm{C}$ for $30 \mathrm{~min}$ in the dark. Images were captured using a fluorescence microscope (magnification, $\mathrm{x} 200$ ) and the mean density was analyzed using ImageJ software version 1.8.0 (National Institutes of Health) using the following formula: Mean density = integrated density/total area. The fluorescence intensity of DCF was measured by flow cytometry (BD LSR II; BD Biosciences) and analyzed using FACSDiva software version 4.1 (BD Biosciences).

TUNEL assay. Apoptosis was analyzed using the One Step TUNEL Apoptosis Assay kit (cat. no. C1090; Beyotime Institute of Biotechnology), according to the manufacturer's instructions. Briefly, H9C2 cells were seeded into a 6-well plate at a density of $5 \times 10^{4}$ cells. Cells were co-treated with Sal B and LPS + ATP (stimulation) as aforementioned. Subsequently, cells were fixed in $4 \%$ paraformaldehyde for $30 \mathrm{~min}$ at $37^{\circ} \mathrm{C}$, permeabilized in $0.1 \%$ Triton $\mathrm{X}-100$ for $2 \mathrm{~min}$ and incubated with TUNEL assay reagents for $1 \mathrm{~h}$ at $37^{\circ} \mathrm{C}$. Images were captured using a fluorescence microscope and fluorescence was measured using a fluorescence spectrophotometer (absorbance wavelength, $550 \mathrm{~nm}$ ). The mean density was determined as previously described above.

Mitochondrial membrane potential (MMP) measurement. An MMP assay kit with JC-1 (cat. no. C2006; Beyotime Institute of Biotechnology) was used to evaluate MMP, according to the manufacturer's protocol. Briefly, 1x106 cells/well were incubated with JC-1 for $30 \mathrm{~min}$ at $37^{\circ} \mathrm{C}$, rinsed twice with PBS and the staining images were captured under a fluorescence microscope. The MMP was indicated by the ratio of red/green fluorescence intensity.

Statistical analysis. Experiments were repeated $\geq 3$ times. Data are presented as the mean \pm standard deviation. The statistically significant differences between the means of multiple groups were analyzed by one-way ANOVA followed by Tukey's multiple comparison test. All statistical analyses were performed using GraphPad Prism 7 software (GraphPad Software, Inc.). $\mathrm{P}<0.05$ was considered to indicate a statistically significant difference. 

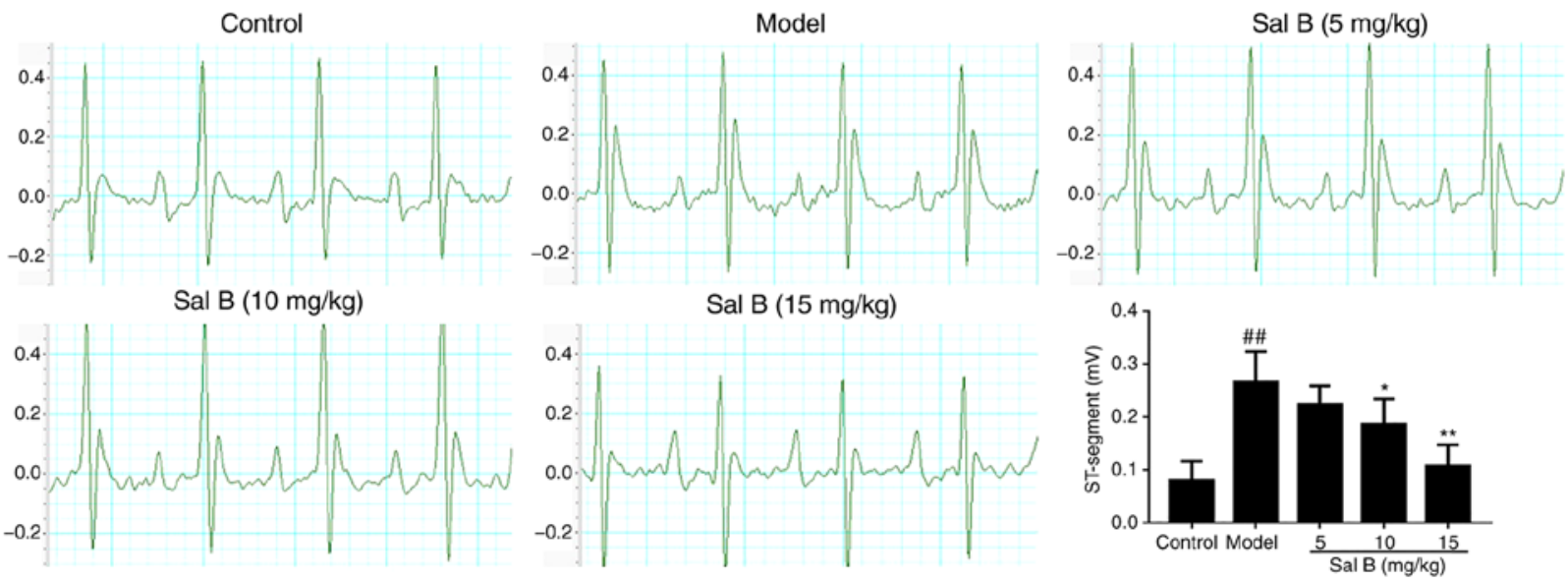

Figure 1. Effects of Sal B on changes to the ST-segment in rats with isoproterenol-induced myocardial ischemia (n=10 rats/group). Sal B, salvianolic acid B ${ }^{\# \#} \mathrm{P}<0.01$ vs. the control group; ${ }^{*} \mathrm{P}<0.05,{ }^{* *} \mathrm{P}<0.01$ vs. the model group.

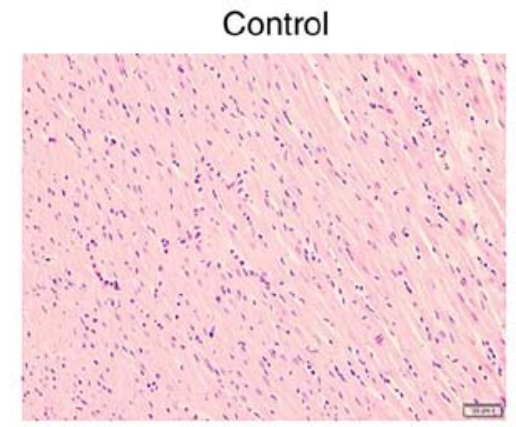

Sal B $(10 \mathrm{mg} / \mathrm{kg})$

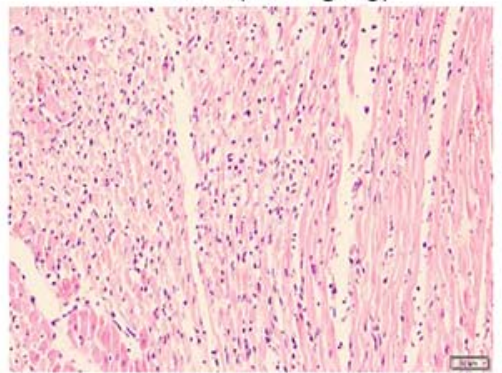

Model

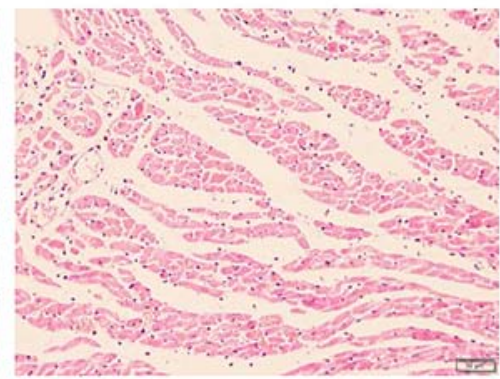

Sal B (15 mg/kg)

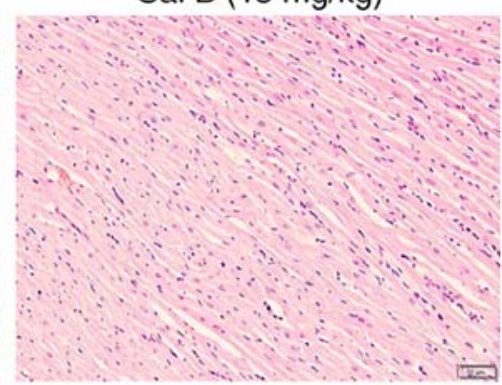

Sal B (5 mg/kg)

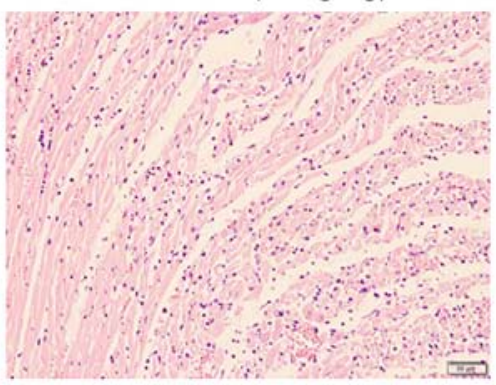

Figure 2. Histopathological observation of hematoxylin and eosin stained hearts in each group of rats ( $\mathrm{n}=3$ rats/group; magnification, $\mathrm{x} 200)$. Sal B, salvianolic acid B.

\section{Results}

Echocardiography of rats with acute myocardial ischemia. Standard echocardiography was performed at room temperature for all groups of rats $24 \mathrm{~h}$ after the administration of Sal B on the 7th day. Control rats exhibited a normal ECG pattern with regular sinus rhythm (Fig. 1). However, an irregular sinus rhythm with significant elevation of the ST-segment was observed in the ECGs of ISO-induced model rats compared with those of control rats. Furthermore, 10 and $15 \mathrm{mg} / \mathrm{kg} \mathrm{Sal} \mathrm{B}$ treatment markedly reversed these ISO-induced alterations (Fig. 1).

Pathological observation. The results of H\&E staining revealed that the hearts of rats in the control group maintained a normal form and function (Fig. 2). However, the hearts of rats in the model group exhibited obvious rupture and lysis of myocardial fibers, accompanied by marked inflammatory cell infiltration. Notably, treatment with Sal B alleviated these pathological observations (Fig. 2).

Sal $B$ reduces the levels of $C K-M B, G O T$ and $I L-1 \beta$ in the serum of rats with myocardial ischemia. Elevation in the serum levels of cardiac markers, such as CK-MB and GOT, is an important basis for the diagnosis of acute myocardial ischemia (27). To evaluate the effects of Sal B on myocardial ischemic injury, the serum levels of CK-MB, GOT and IL-1 $\beta$ were determined. As shown in Fig. 3, intraperitoneal injection of ISO significantly increased the levels of CK-MB, GOT and IL-1 $\beta$ compared with those in the control group; treatment with Sal B $(5,10$ and $15 \mathrm{mg} / \mathrm{kg})$ significantly reversed the levels of these factors. 

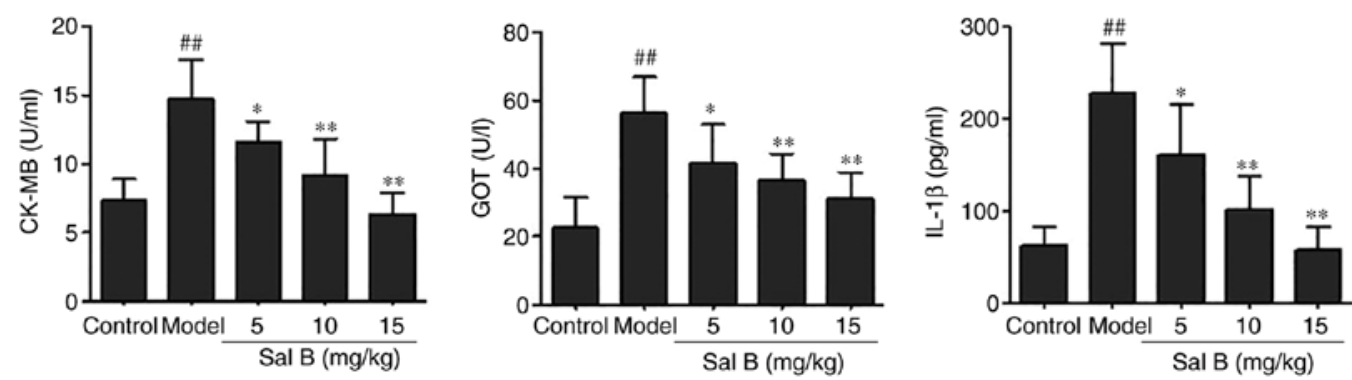

Figure 3. Sal B reduces the levels of CK-MB, GOT and IL-1 $\beta$ in myocardial ischemia model rat blood serum samples ( $\mathrm{n}=10$ rats/group). ${ }^{\# \#} \mathrm{P}<0.01 \mathrm{vs.}$. the control group; " $\mathrm{P}<0.05,{ }^{* * *} \mathrm{P}<0.01$ vs. the model group. CK-MB, creatine kinase isoenzyme MB; GOT, glutamic oxaloacetic transaminase; IL-1 $\beta$, interleukin- $1 \beta$; Sal B, salvianolic acid B.
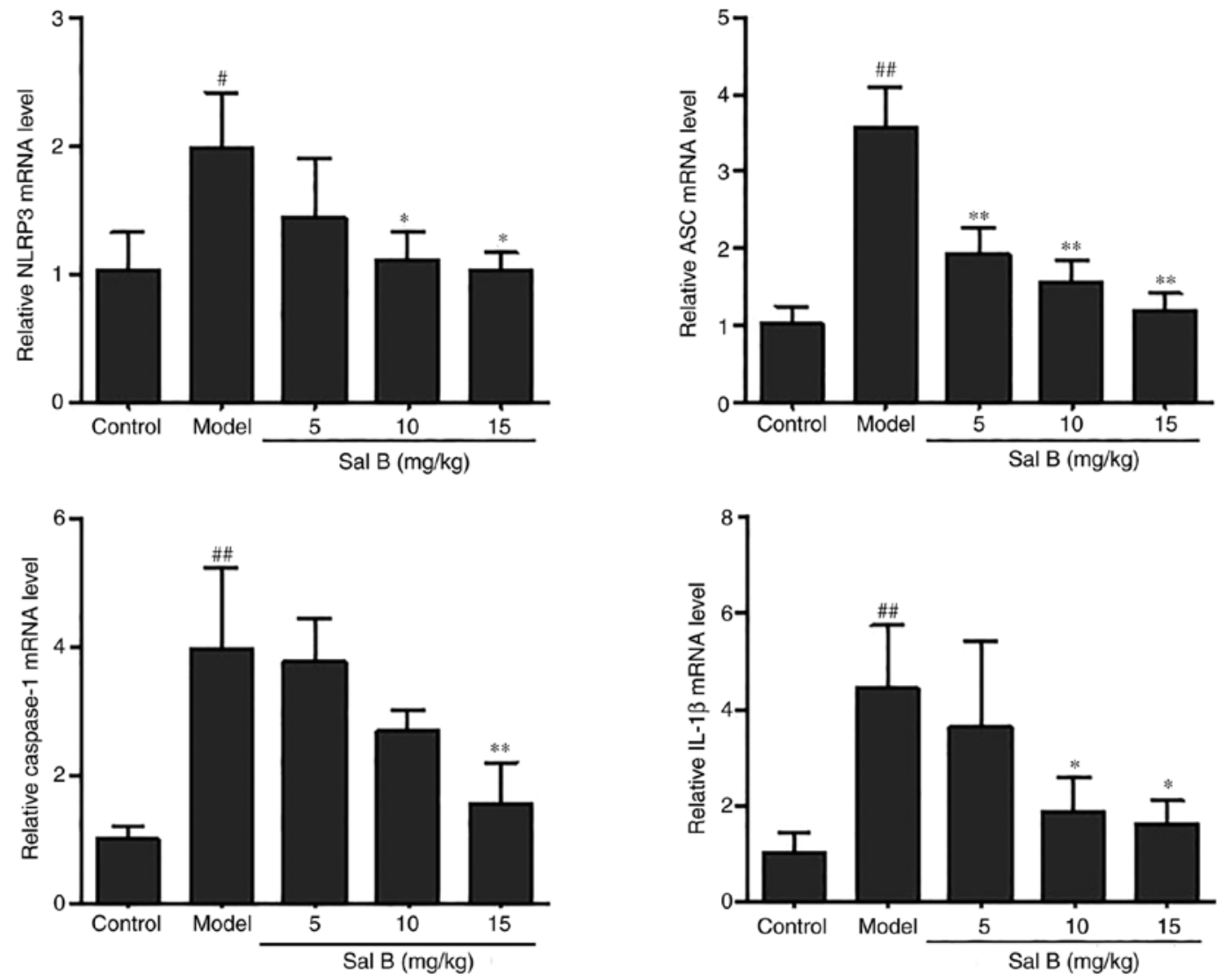

Figure 4. Sal B treatment significantly reduces the mRNA expression levels of NLRP3, ASC, caspase-1 and IL-1 $\beta$ in rat hearts as detected by reverse transcription-quantitative PCR analysis ( $\mathrm{n}=3$ rats/group). The mRNA expression levels were normalized to those of GAPDH. ${ }^{\#} \mathrm{P}<0.05,{ }^{\# \#} \mathrm{P}<0.01$ vs. the control group; " $\mathrm{P}<0.05,{ }^{* *} \mathrm{P}<0.01$ vs. the model group. ASC, apoptosis-associated speck like protein; NLRP3, NLR family pyrin domain-containing 3 ; Sal B, salvianolic acid B.

Effects of Sal B on the mRNA expression levels of NLRP3, $A S C$, caspase- 1 and $I L-1 \beta$ in rats with ISO-induced myocardial ischemia. To investigate whether Sal B could inhibit the expression of the NLRP3 inflammasome in rats with ISO-induced myocardial ischemia, RT-qPCR analysis was conducted to determine the mRNA expression levels of the related genes. ISO stimulation significantly increased the mRNA expression levels of the NLRP3 inflammasome components compared with those in the control group (Fig. 4). However, the expression levels of NLRP3, ASC, caspase- 1 and IL-1 $\beta$ in the Sal B-treated group $(15 \mathrm{mg} / \mathrm{kg})$ were significantly reduced compared with those in the model group (Fig. 4).
Sal B treatment promotes SIRTI-mediated mitophagy. The contraction and functioning of the heart are mainly dependent on mitochondria to provide energy. A previous study demonstrated that myocardial ischemic injury is often accompanied by mitochondrial dysfunction (28). Mitophagy is considered an effective process to remove damaged mitochondria and maintain normal mitochondrial function (29). To investigate the effect of Sal B on mitophagy during myocardial ischemic injury in cardiomyocytes, the expression levels of the proteins SIRT1, PINK1 and Parkin and were detected in H9C2 cells (Fig. 5). The differences in the protein expression levels of SIRT1 and Parkin in the control and LPS + ATP groups were not statistically significant; however, the expression levels 

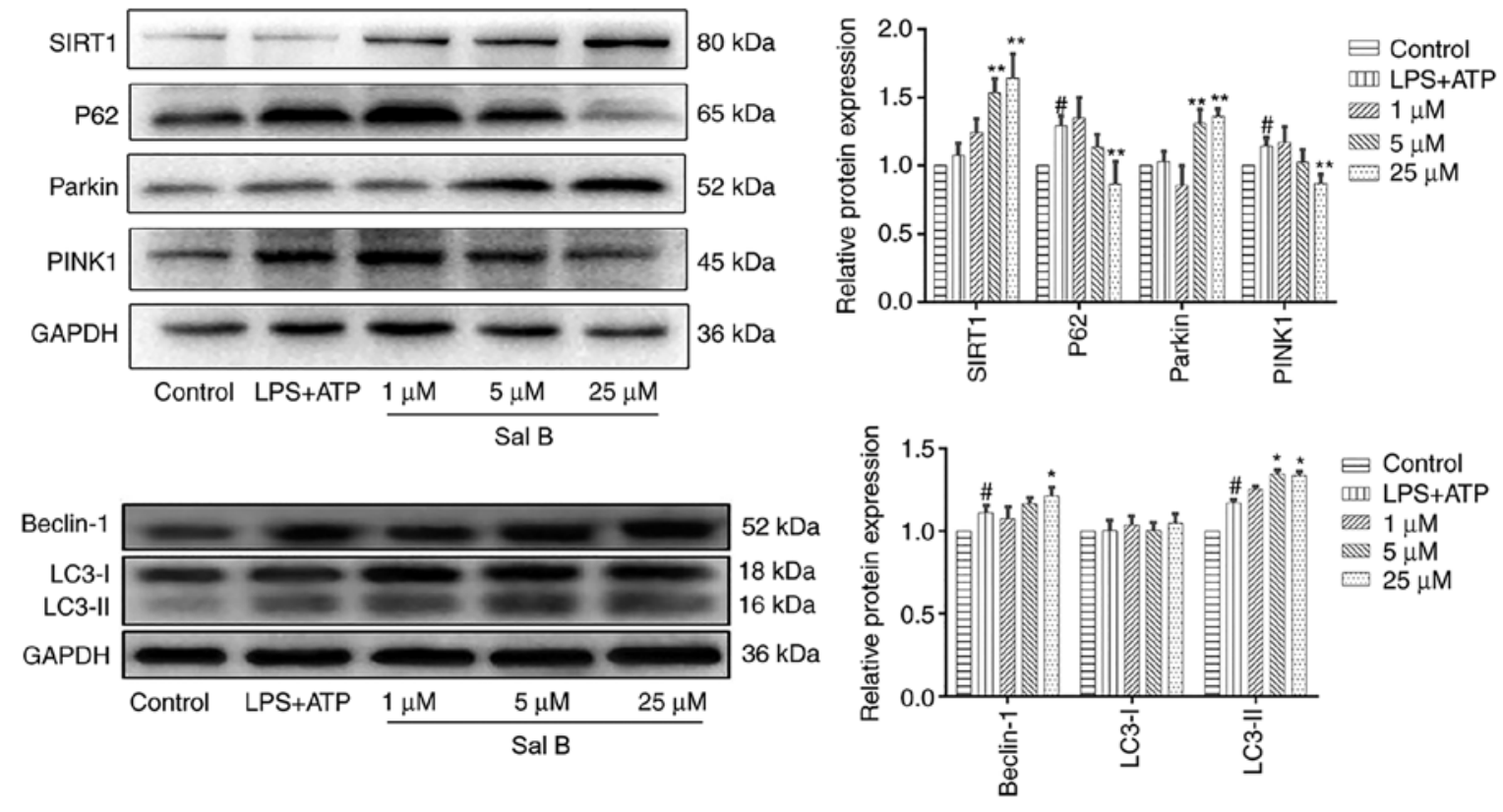

Figure 5. Sal B treatment significantly promotes mitophagy in $\mathrm{H} 9 \mathrm{C} 2$ cells. Western blot analysis was performed to determine the protein expression levels of SIRT1, P62, Parkin, PINK1, Beclin-1 and LC3 in each group. The protein expression levels were normalized to those of GAPDH. Data are presented as the mean \pm standard deviation from three independent experiments. ${ }^{~} \mathrm{P}<0.05$ vs. the control group; ${ }^{*} \mathrm{P}<0.05,{ }^{* *} \mathrm{P}<0.01$ vs. the LPS + ATP group. ATP, adenosine triphosphate; LC3, microtubule-associated protein 1A/1B-light chain 3; LPS, lipopolysaccharide; PINK1, PTEN-induced kinase 1; Sal B, salvianolic acid B; SIRT1, sirtuin 1.
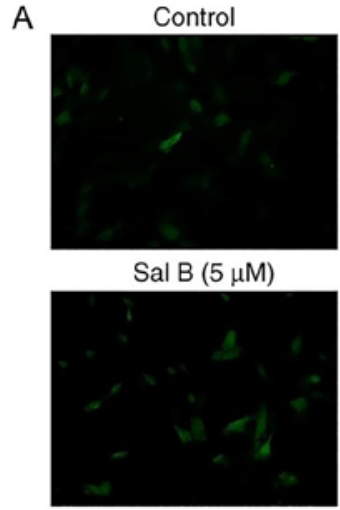

B

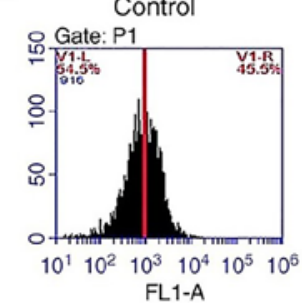

LPS+ATP

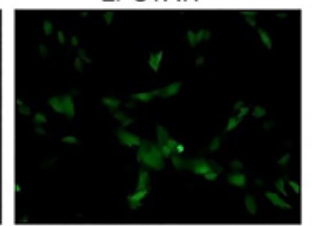

Sal B $(25 \mu \mathrm{M})$

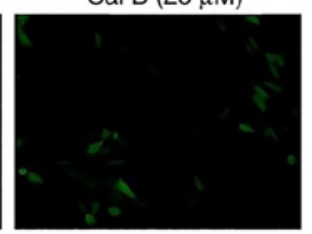

LPS+ATP

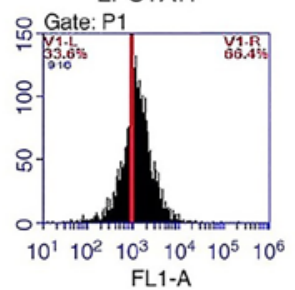

Sal B $(1 \mu \mathrm{M})$
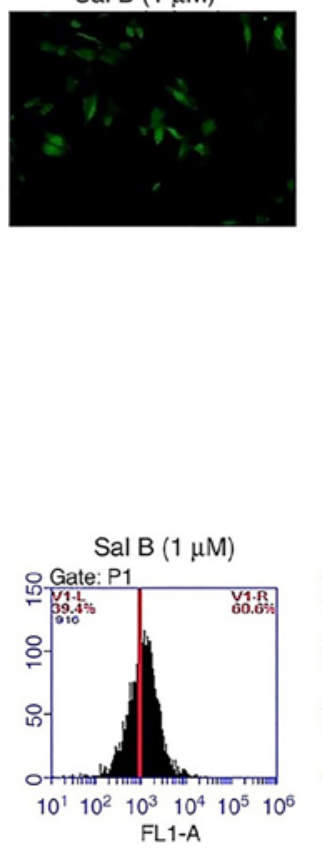
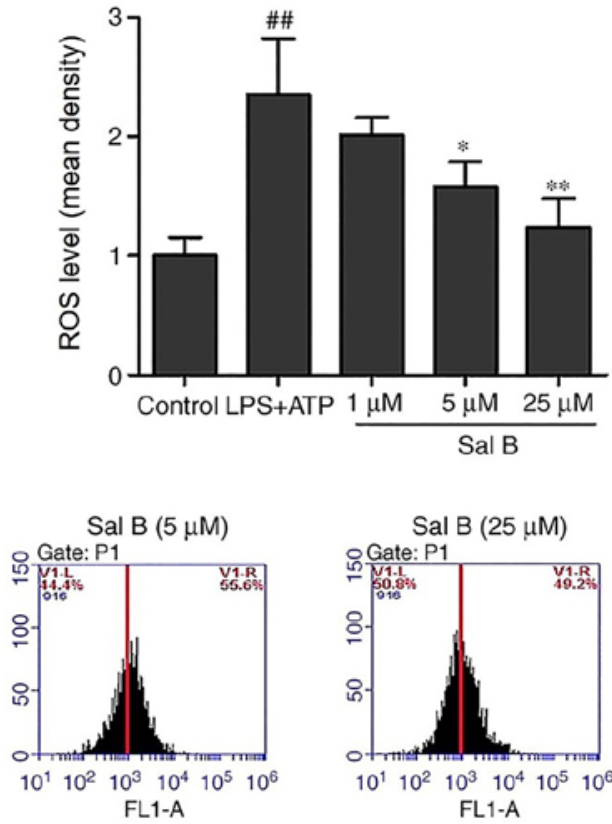

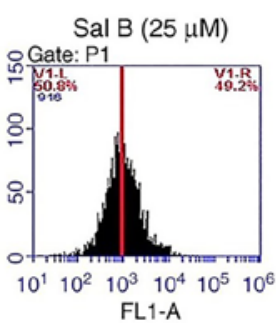

Figure 6. Effects of Sal B treatment on the LPS + ATP-induced intracellular accumulation of ROS in H9C2 cells (n=3). (A) Fluorescence microscopy showed that Sal B treatment (5 and $25 \mu \mathrm{M}$ ) significantly reduced the intracellular ROS levels in $\mathrm{H} 9 \mathrm{C} 2$ cells (magnification, x200). (B) Flow cytometry analysis of the fluorescence intensity of dichlorofluorescein. ${ }^{\sharp \#} \mathrm{P}<0.01$ vs. the control group; ${ }^{*} \mathrm{P}<0.05,{ }^{* *} \mathrm{P}<0.01$ vs. the LPS + ATP group. ATP, adenosine triphosphate; LPS, lipopolysaccharide; ROS, reactive oxygen species; Sal B, salvianolic acid B.

of SIRT1 and Parkin were significantly decreased in the LPS + ATP group compared with those in the Sal B-treated groups $(5$ and $25 \mu \mathrm{M})$. Furthermore, LPS + ATP stimulation significantly increased the expression levels of PINK1 compared with the control group; however, Sal B treatment $(25 \mu \mathrm{M})$ significantly reduced the expression levels. In addi- tion, the expression levels of autophagy-related proteins P62, LC3 and Beclin-1 were evaluated. Notably, LPS + ATP stimulation significantly increased the expression level of autophagy substrate P62 compared with the control group; however, Sal B treatment $(25 \mu \mathrm{M})$ significantly reduced the expression levels. Furthermore, LPS + ATP significantly upregulated the 


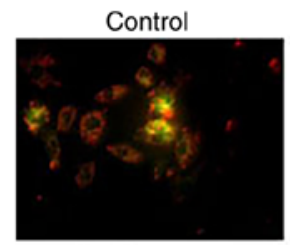

Sal B $(5 \mu \mathrm{M})$

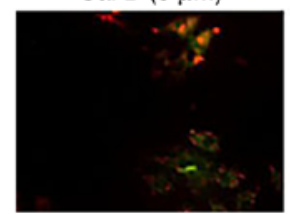

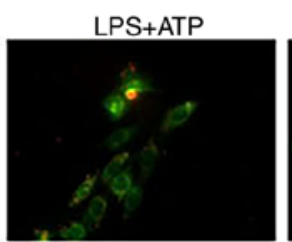

Sal B $(25 \mu \mathrm{M})$

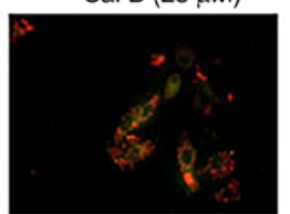

Sal B $(1 \mu \mathrm{M})$
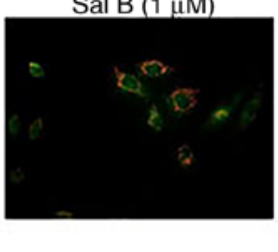

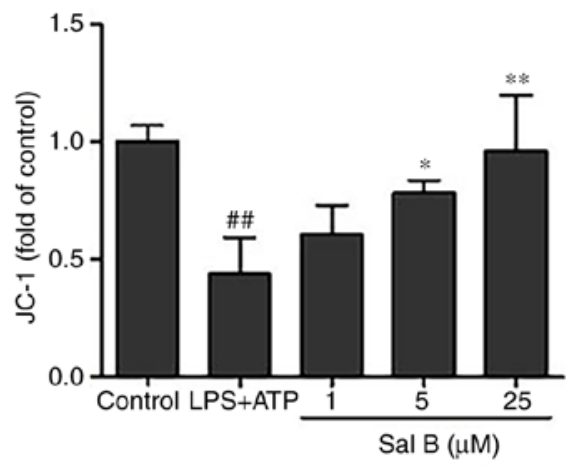

Figure 7. Effect of Sal B treatment on mitochondrial membrane potential in LPS + ATP-induced H9C2 cells ( $\mathrm{n}=3$; magnification, $\mathrm{x} 200)$. ${ }^{\#} \mathrm{P}<0.01$ vs. the control group; ${ }^{*} \mathrm{P}<0.05,{ }^{* *} \mathrm{P}<0.01$ vs. the LPS + ATP group. ATP, adenosine triphosphate; LPS, lipopolysaccharide; Sal B, salvianolic acid B.
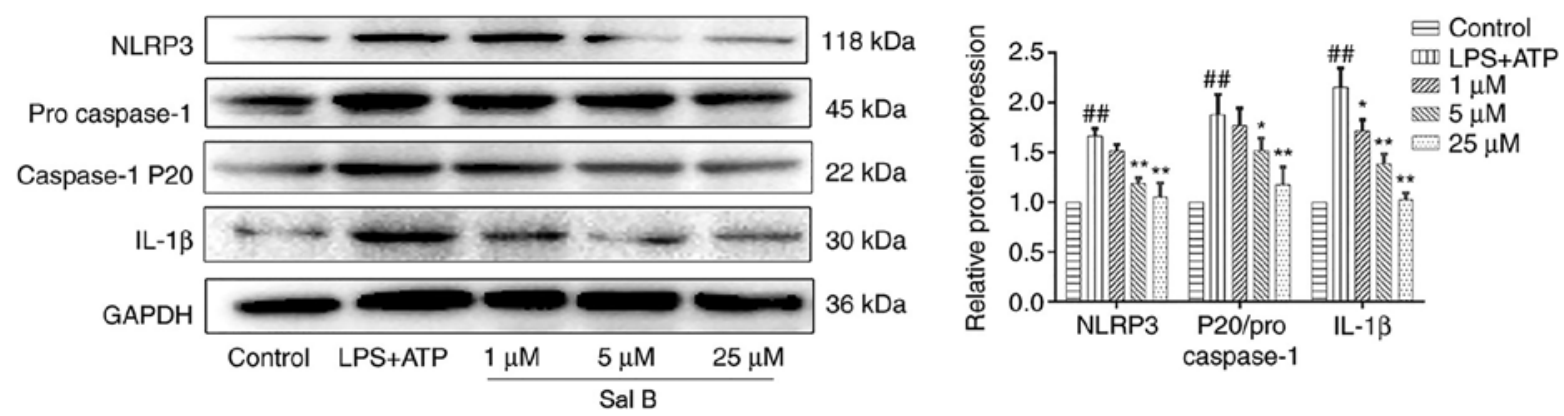

Figure 8. Sal B treatment significantly reduces the protein expression levels of NLRP3, caspase-1 and IL-1 $\beta$ in LPS + ATP-induced H9C2 cells. The expression levels were normalized to those of GAPDH. Data are presented as the mean \pm standard deviation from three independent experiments. ${ }^{\# \prime} \mathrm{P}<0.01 \mathrm{vs}$. the control group; "P $<0.05,{ }^{* *} \mathrm{P}<0.01$ vs. the LPS + ATP group. ATP, adenosine triphosphate; LPS, lipopolysaccharide; NLRP3, NLR family pyrin domain-containing 3; Sal B, salvianolic acid B.
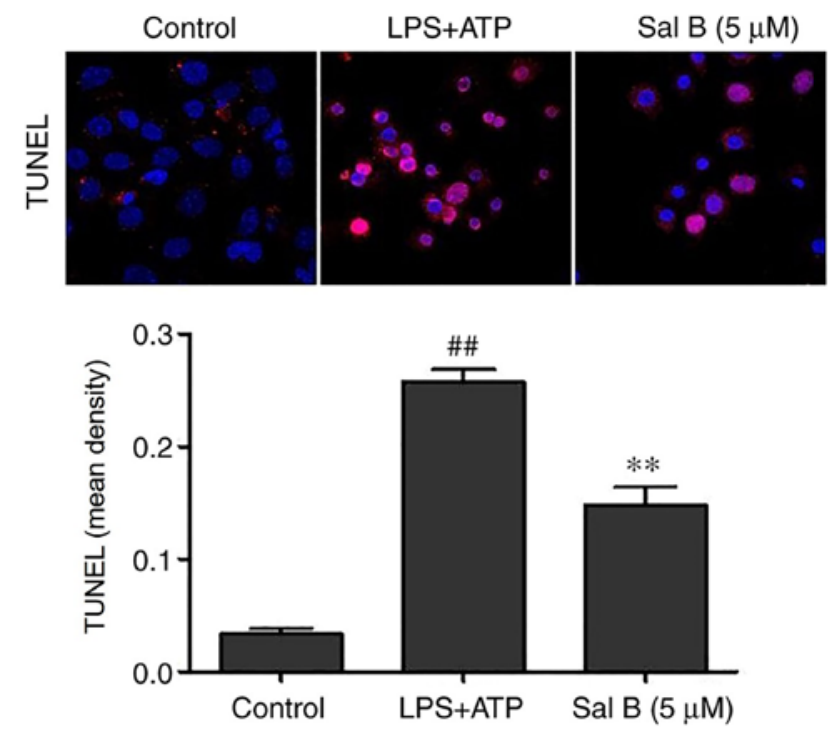

Figure 9. Cell apoptosis was determined by TUNEL assay in Sal B treated, LPS + ATP-induced $\mathrm{H} 9 \mathrm{C} 2$ cells $(\mathrm{n}=3$; magnification, $\mathrm{x} 200){ }^{* * *} \mathrm{P}<0.01$ vs. the LPS + ATP group; ${ }^{\# \#} \mathrm{P}<0.01$ vs. the control group. ATP, adenosine triphosphate; LPS, lipopolysaccharide; IOD, integrated optical density.

expression levels of cellular autophagy markers Beclin-1 and LC3-II compared with those in the control group. The expression levels of Beclin-1 following Sal B treatment $(25 \mu \mathrm{M})$ and LC3-II following Sal B treatment (5 and $25 \mu \mathrm{M}$ ) were signifi- cantly increased compared with those in LPS + ATP group, thus suggesting that Sal B $(25 \mu \mathrm{M})$ may mediate the induction of mitophagy and autophagy in myocardial ischemic injury.

Sal B inhibits LPS + ATP-induced ROS production. Excessive ROS production has been associated with impaired mitochondrial function and increased $\mathrm{H} 9 \mathrm{C} 2$ cell apoptotic rate (30). As shown in the fluorescence images in Fig. 6, LPS + ATP stimulation significantly increased the fluorescence intensity compared with the control group, indicating the production of ROS. Conversely, pretreatment with Sal B attenuated the DCF fluorescence induced by LPS + ATP. Consistent with the fluorescence results, flow cytometric analysis demonstrated that the levels of intracellular ROS production in the LPS + ATP group were notably increased compared with those in the control group, whereas pretreatment with Sal B reversed this effect (Fig. 6).

Effects of Sal B on MMP. A previous study reported that decreased MMP is closely associated with mitochondrial dysfunction (31). To investigate whether Sal B could inhibit mitochondrial dysfunction by increasing MMP, a JC-1 kit was used. The JC-1 red/green fluorescence ratio was used as a parameter to estimate changes in MMP. The results revealed that the MMP in $\mathrm{H} 9 \mathrm{C} 2$ cells was significantly reduced by LPS + ATP stimulation compared with in the control group, whereas Sal B pre-treatment (5 and $25 \mu \mathrm{M}$ ) significantly increased its potential (Fig. 7). 
Effects of Sal B on activation of the NLRP3 inflammasome. A previous study suggested that the occurrence of mitophagy may lead to degradation of the NLRP3 inflammasome, which in turns prevents the activation of NLRP3 inflammasome signaling and reduces the secretion of mature IL-1 $\beta$ (32). To explore whether Sal B treatment could attenuate the expression levels of the NLRP3 inflammasome components, the protein expression levels of NLRP3, caspase-1 and IL-1 $\beta$ were evaluated. The results demonstrated that the protein expression levels of NLRP3, caspase- 1 and IL-1 $\beta$ were significantly increased in $\mathrm{H} 9 \mathrm{C} 2$ cells following LPS + ATP stimulation; however, pre-treatment with Sal B $(25 \mu \mathrm{M})$ significantly reduced the expression levels of these proteins (Fig. 8).

Effects of Sal B on the apoptosis of $\mathrm{H} 9 \mathrm{C} 2$ cells. Assembly of the NLRP3 inflammasome may activate caspase- 1 and promote apoptosis (33). As shown in Fig. 9, LPS + ATP stimulation significantly promoted apoptosis compared with in the control group, whereas Sal B pre-treatment $(5 \mu \mathrm{M})$ significantly reversed this effect.

\section{Discussion}

In the present study, Sal B markedly attenuated ISO-induced acute myocardial ischemic injury in rats and reduced the serum levels of several cytokines, including CK-MB, GOT and IL-1 $\beta$. Furthermore, to the best of our knowledge, the present study was the first to demonstrate that Sal B treatment may be able to maintain mitochondrial function, promote SIRT1-mediated mitophagy and inhibit activation of the NLRP3 inflammasome. However, the present study did not provide much information on cardiac pathology and function; we aim to assess these further in future experiments.

It is widely accepted that myocardial ischemia is often accompanied by severe inflammatory response $(34,35)$. As the core target of inflammatory responses, the NLRP 3 inflammasome has been considered the key regulator of numerous inflammatory diseases (36). LPS + ATP is a classical activator of the NLRP3 inflammasome; it is widely used in studies of NLRP3 inflammasome-related diseases, including myocardial ischemia $(37,38)$. Activation of the classic NLRP3 inflammasome requires two steps: Firstly, microorganisms or other inflammatory factors bind to Toll-like receptor 4 (TLR4) and activate the TLR4/NF- $\mathrm{KB}$ signaling pathway to induce the expression of NLRP3, pro-caspase-1 and pro-IL-1 $\beta$ (priming phase). Secondly, extracellular ATP or other stimuli, such as lysosome destabilization and mitochondrial dysfunction (39), directly stimulate the activation of caspase-1, thereby leading to the release of IL- $1 \beta$ and IL-18 (triggering phase). Activation of the NLRP3 inflammasome in cardiomyocytes requires the involvement of both priming and triggering phases (40). A previous study in our laboratory reported that $\mathrm{Sal} \mathrm{B}$ exerted its cardioprotective effects by inhibiting the TLR4/NF- $\mathrm{KB} / \mathrm{NLRP} 3$ signaling pathway (41), and myeloid differential protein-2 was considered the potential target. However, owing to the multi-target characteristics of natural products, it was hypothesized that Sal B may inhibit the NLRP3 inflammasome through other mechanisms. Therefore, the present study explored whether Sal B could inhibit the triggering phase of the NLRP3 inflammasome, thereby attenuating myocardial ischemic injury.

The contraction and functioning of the heart are mainly dependent on mitochondria to provide energy. Emerging evidence has suggested that the accumulation of dysfunctional mitochondria may result in increased intracellular ROS, thus leading to cardiac dysfunction (42). Mitophagy is an effective process that can remove damaged mitochondria and maintain mitochondrial function (43). The association between SIRT1 and mitophagy has also been previously reported. For example, it has been reported that the SIRT1-PINK1-Parkin axis may participate in resveratrol-induced mitophagy and cardioprotection (44). Furthermore, it has been revealed that promoting mitophagy may negatively regulate activation of the NLRP3 inflammasome, which may exert significant protective effects against myocardial ischemia $(45,46)$. In conclusion, the present study demonstrated that Sal B could significantly inhibit intracellular ROS production, increase the mitochondrial membrane potential and regulate the protein expression levels of SIRT1, Parkin and PINK1 in myocardial ischemia, which in turn could mediate the promotion of mitophagy and inactivation of the NLRP3 inflammasome. Therefore, Sal B, as a natural product with little toxicity, may provide a novel therapeutic strategy for the treatment of myocardial ischemia.

\section{Acknowledgements}

Not applicable.

\section{Funding}

The present study was supported by The Jiangsu Administration of Traditional Chinese Medicine (grant no. ZD301701).

\section{Availability of data and materials}

The datasets used and/or analyzed during the current study are available from the corresponding author on reasonable request.

\section{Authors' contributions}

YH and XW contributed equally to this work. LX designed this project. YH, XW, YP and QL performed the experiments and analyzed the data. YH and XW contributed to the writing of the manuscript. All authors contributed to the revision of this manuscript, and read and approved the final manuscript.

\section{Ethics approval and consent to participate}

The protocol was approved by the Animal Ethics Committee of Nanjing University of Chinese Medicine (approval no. 201906A036).

\section{Patient consent for publication}

Not applicable.

\section{Competing interests}

The authors declare that they have no competing interests. 


\section{References}

1. Chen WW, Gao RL, Liu LS, Zhu ML, Wang W, Wang YJ, Wu ZS, Li HJ, Gu DF, Yang YJ, et al: China cardiovascular diseases report 2015: A summary. J Geriatr Cardiol 14: 1-10, 2017.

2. Kang PF, Wu WJ, Tang Y, Xuan L, Guan SD, Tang B, Zhang H, Gao Q and Wang HJ: Activation of ALDH2 with low concentration of ethanol attenuates myocardial ischemia/reperfusion injury in diabetes rat model. Oxid Med Cell Longev 2016 : 6190504, 2016

3. Cao DJ, Schiattarella GG, Villalobos E, Jiang N, May HI, Li T, Chen ZJ, Gillette TG and Hill JA: Cytosolic DNA sensing promotes macrophage transformation and governs myocardial ischemic injury. Circulation 137: 2613-2634, 2018.

4. Jiang S, Liu Y, Wang J, Zhang Y, Rui Y, Zhang Y and Li T: Cardioprotective effects of monocyte locomotion inhibitory factor on myocardial ischemic injury by targeting vimentin. Life Sci 167: 85-91, 2016

5. Zhu L, Wei T, Gao J, Chang X, He H, Luo F, Zhou R, Ma C, Liu Y and Yan T: The cardioprotective effect of salidroside against myocardial ischemia reperfusion injury in rats by inhibiting apoptosis and inflammation. Apoptosis 20: 1433-1443, 2015.

6. Marchetti C, Toldo S, Chojnacki J, Mezzaroma E, Liu K, Salloum FN, Nordio A, Carbone S, Mauro AG, Das A, et al: Pharmacologic inhibition of the NLRP3 inflammasome preserves cardiac function after ischemic and nonischemic injury in the mouse. J Cardiovasc Pharmacol 66: 1-8, 2015.

7. Swanson KV, Deng M and Ting JP: The NLRP3 inflammasome: Molecular activation and regulation to therapeutics. Nat Rey Immunol 19: 477-489, 2019.

8. He Q, Li Z, Meng C, Wu J, Zhao Y and Zhao J: Parkin-dependent mitophagy is required for the inhibition of ATF4 on NLRP3 inflammasome activation in cerebral ischemia-reperfusion injury in rats. Cells 82019

9. Wan Z, Fan Y, Liu X, Xue J, Han Z, Zhu C and Wang X: NLRP3 inflammasome promotes diabetes-induced endothelial inflammation and atherosclerosis. Diabetes Metab Syndr Obes 12: 1931-1942, 2019.

10. Mao L, Kitani A, Strober W and Fuss IJ: The role of NLRP3 and IL-1 $\beta$ in the pathogenesis of inflammatory bowel disease. Front Immunol 9: 2566, 2018.

11. Toldo S, Marchetti C, Mauro AG, Chojnacki J, Mezzaroma E, Carbone S, Zhang S, Van Tassell B, Salloum FN and Abbate A: Inhibition of the NLRP3 inflammasome limits the inflammatory injury following myocardial ischemia-reperfusion in the mouse. Int J Cardiol 209: 215-220, 2016.

12. Han Y, Luo H, Wang H, Cai J and Zhang Y: SIRT1 induces resistance to apoptosis in human granulosa cells by activating the ERK pathway and inhibiting NF- $\mathrm{B}$ B signaling with anti-inflammatory functions. Apoptosis 22: 1260-1272, 2017.

13. Wong WT, Li LH, Rao YK, Yang SP, Cheng SM, Lin WY, Cheng CC, Chen A and Hua KF: Repositioning of the beta-blocker carvedilol as a novel autophagy inducer that inhibits the NLRP3 inflammasome. Front Immunol 9: 1920, 2018.

14. Akkafa F, Halil Altiparmak I, Erkus ME, Aksoy N, Kaya C, Ozer A, Sezen H, Oztuzcu S, Koyuncu I and Umurhan B: Reduced SIRT1 expression correlates with enhanced oxidative stress in compensated and decompensated heart failure. Redox Biol 6: 169-173, 2015

15. Wang L, Ma R, Liu C, Liu H, Zhu R, Guo S, Tang M, Li Y, Niu J, Fu M, et al: Salvia miltiorrhiza: A potential red light to the development of cardiovascular diseases. Curr Pharm Des 23 : 1077-1097, 2017

16. Han F, Xing RH, Chen LQ, Chen L, Xiong W, Yang M and Zhao ZD: Research progress of anti-drug resistance in traditional Chinese medicine. Zhongguo Zhong Yao Za Zhi 41: 813-817, 2016 (In Chinese)

17. Gong L, Di C, Xia X, Wang J, Chen G, Shi J, Chen P, Xu H and Zhang W: AKT/mTOR signaling pathway is involved in salvianolic acid B-induced autophagy and apoptosis in hepatocellular carcinoma cells. Int J Oncol 49: 2538-2548, 2016.

18. Lou Y, Wang C, Zheng W, Tang Q, Chen Y, Zhang X, Guo X and Wang J: Salvianolic acid B inhibits IL-1 $\beta$-induced inflammatory cytokine production in human osteoarthritis chondrocytes and has a protective effect in a mouse osteoarthritis model. Int Immunopharmacol 46: 31-37, 2017.

19. Liu X, Xavier C, Jann J and Wu H: Salvianolic acid B (Sal B) protects retinal pigment epithelial cells from oxidative stress-induced cell death by activating glutaredoxin 1 (Grx1). Int J Mol Sci 17: 1835, 2016
20. Li D, Wang J, Hou J, Fu J, Liu J and Lin R: Salvianolic acid B induced upregulation of miR-30a protects cardiac myocytes from ischemia/reperfusion injury. BMC Complement Altern Med 16: 336, 2016.

21. Dhivya V, Priya LB, Chirayil HT, Sathiskumar S, Huang CY and Padma VV: Piperine modulates isoproterenol induced myocardial ischemia through antioxidant and anti-dyslipidemic effect in male Wistar rats. Biomed Pharmacother 87: 705-713, 2017.

22. Ke Z, Wang G, Yang L, Qiu H, Wu H, Du M, Chen J, Song J, Jia X and Feng L: Crude terpene glycoside component from Radix paeoniae rubra protects against isoproterenol-induced myocardial ischemic injury via activation of the PI3K/AKT/mTOR signaling pathway. J Ethnopharmacol 206: 160-169, 2017.

23. Livak KJ and Schmittgen TD: Analysis of relative gene expression data using real-time quantitative PCR and the 2(-Delta Delta C(T)) Method. Methods 25: 402-408, 2001.

24. Huang X, Zuo L, Lv Y, Chen C, Yang Y, Xin H, Li Y and Qian Y: Asiatic acid attenuates myocardial ischemia/reperfusion injury via $A k t / G S K-3 \beta / H I F-1 \alpha$ signaling in rat $\mathrm{H} 9 \mathrm{c} 2$ cardiomyocytes. Molecules 21: 21,2016.

25. Li P, Lin N, Guo M, Huang $\mathrm{H}, \mathrm{Yu} \mathrm{T}$ and Zhang L: REDD1 knockdown protects $\mathrm{H} 9 \mathrm{c} 2$ cells against myocardial ischemia/reperfusion injury through $\mathrm{Akt} / \mathrm{mTORC} 1 / \mathrm{Nrf} 2$ pathway-ameliorated oxidative stress: An in vitro study. Biochem Biophys Res Commun 519: 179-185, 2019.

26. Zou J, Zhang Y, Sun J, Wang X, Tu H, Geng S, Liu R, Chen Y and $\mathrm{Bi} \mathrm{Z}$ : Deoxyelephantopin induces reactive oxygen species-mediated apoptosis and autophagy in human osteosarcoma cells. Cell Physiol Biochem 42: 1812-1821, 2017.

27. Zhang X, Du Q, Yang Y, Wang J, Dou S, Liu C and Duan J: The protective effect of Luteolin on myocardial ischemia/reperfusion (I/R) injury through TLR4/NF- $\mathrm{KB} / \mathrm{NLRP} 3$ inflammasome pathway. Biomed Pharmacother 91: 1042-1052, 2017.

28. Xue W, Wang X, Tang H, Sun F, Zhu H, Huang D and Dong L: Vitexin attenuates myocardial ischemia/reperfusion injury in rats by regulating mitochondrial dysfunction induced by mitochondrial dynamics imbalance. Biomed Pharmacother 124 109849,2020

29. Youle RJ and Narendra DP: Mechanisms of mitophagy. Nat Rev Mol Cell Biol 12: 9-14, 2011.

30. Wang M, Wang R, Xie X, Sun G and Sun X: Araloside C protects $\mathrm{H} 9 \mathrm{c} 2$ cardiomyoblasts against oxidative stress via the modulation of mitochondrial function. Biomed Pharmacother 117: 109143, 2019.

31. Wu C, Zhao W, Yu J, Li S, Lin L and Chen X: Induction of ferroptosis and mitochondrial dysfunction by oxidative stress in PC12 cells. Sci Rep 8: 574, 2018.

32. Kim MJ, Yoon JH and Ryu JH: Mitophagy: A balance regulator of NLRP3 inflammasome activation. BMB Rep 49: 529-535, 2016.

33. He Y, Hara H and Núñez G: Mechanism and regulation of NLRP3 inflammasome activation. Trends Biochem Sci 41: 1012-1021, 2016

34. Hammadah M, Sullivan S, Pearce B, Al Mheid I, Wilmot K, Ramadan R, Tahhan AS, O'Neal WT, Obideen M, Alkhoder A, et al: Inflammatory response to mental stress and mental stress induced myocardial ischemia. Brain Behav Immun 68: 90-97, 2018.

35. Hansson GK: Inflammation, atherosclerosis, and coronary artery disease. N Engl J Med 352: 1685-1695, 2005.

36. Mangan MSJ, Olhava EJ, Roush WR, Seidel HM, Glick GD and Latz E: Targeting the NLRP3 inflammasome in inflammatory diseases. Nat Rev Drug Discov 17: 588-606, 2018.

37. Wang DS, Yan LY, Yang DZ, Lyu Y, Fang LH, Wang SB and Du GH: Formononetin ameliorates myocardial ischemia/reperfusion injury in rats by suppressing the ROS-TXNIP-NLRP3 pathway. Biochem Biophys Res Commun 525: 759-766, 2020

38. Yuan X, Juan Z, Zhang R, Sun X, Yan R, Yue F, Huang Y, Yu J and Xia X: Clemastine fumarate protects against myocardial ischemia reperfusion injury by activating the TLR4/PI3K/Akt signaling pathway. Front Pharmacol 11: 28, 2020.

39. Gong Z, Pan J, Shen Q, Li M and Peng Y: Mitochondrial dysfunction induces NLRP3 inflammasome activation during cerebral ischemia/reperfusion injury. J Neuroinflammation 15: 242,2018

40. Latz E, Xiao TS and Stutz A: Activation and regulation of the inflammasomes. Nat Rev Immunol 13: 397-411, 2013.

41. Hu Y, Li Q, Pan Y and Xu L: Sal B alleviates myocardial ischemic injury by inhibiting TLR4 and the priming phase of NLRP3 inflammasome. Molecules 242019. 
42. Zhu W, Liu F, Wang L, Yang B, Bai Y, Huang Y, Li Y, Li W, Yuan Y, Chen C, et al: pPolyHb protects myocardial H9C2 cells against ischemia-reperfusion injury by regulating the Pink1-Parkin-mediated mitochondrial autophagy pathway. Artif Cells Nanomed Biotechnol 47: 1248-1255, 2019.

43. Villanueva Paz M, Cotán D, Garrido-Maraver J, Cordero MD, Oropesa-Ávila M, de La Mata M, Delgado Pavón A, de Lavera I, Alcocer-Gómez E and Sánchez-Alcázar JA: Targeting autophagy and mitophagy for mitochondrial diseases treatment. Expert Opin Ther Targets 20: 487-500, 2016.

44. Das S, Mitrovsky G, Vasanthi HR and Das DK: Antiaging properties of agrape-derived antioxidant are regulated by mitochondrial balance of fusion and fission leading to mitophagy triggered by a signaling network of Sirt1-Sirt3-Foxo3-PINK1-PARKIN. Oxid Med Cell Longev 2014: 345105, 2014.
45. Mai CT, Wu MM, Wang CL, Su ZR, Cheng YY and Zhang XJ: Palmatine attenuated dextran sulfate sodium (DSS)-induced colitis via promoting mitophagy-mediated NLRP3 inflammasome inactivation. Mol Immunol 105: 76-85, 2019.

46. Cao S, Shrestha S, Li J, Yu X, Chen J, Yan F, Ying G, Gu C, Wang L and Chen G: Melatonin-mediated mitophagy protects against early brain injury after subarachnoid hemorrhage through inhibition of NLRP3 inflammasome activation. Sci Rep 7: 2417, 2017.

(i) (5) This work is licensed under a Creative Commons Attribution-NonCommercial-NoDerivatives 4.0 International (CC BY-NC-ND 4.0) License. 